\title{
Téoros
}

Revue de recherche en tourisme

\section{Tenir compte du touriste dans l'accueil d'un musée : l'expérience du Musée de la civilisation à Québec}

\section{Marilyn Johnson}

Volume 11, numéro 2, juillet 1992

Quand les musées s’ouvrent au tourisme...

URI : https://id.erudit.org/iderudit/1078089ar

DOI : https://doi.org/10.7202/1078089ar

Aller au sommaire du numéro

Éditeur(s)

Université du Québec à Montréal

ISSN

0712-8657 (imprimé)

1923-2705 (numérique)

Découvrir la revue

Citer cet article

Johnson, M. (1992). Tenir compte du touriste dans l'accueil d'un musée : l'expérience du Musée de la civilisation à Québec. Téoros, 11(2), 31-33.

https://doi.org/10.7202/1078089ar d'utilisation que vous pouvez consulter en ligne. 


\section{Tenir compte du touriste dans l'accueil d'un musée : l'expérience du Musée de la civilisation à Québec}

Il y a lieuau préalable de souligner certains faits sur le Musée de la civilisation, sa création, son concept et sa mission, ainsi que sur ses orientations. Nous parlerons ensuite de ses publics (visiteurs-touristes) et de ses préoccupations quant aux différentes façons de répondre aux besoins et attentes spécifiques de tous ses visiteurs.

\section{Le Musée de la civilisation: un monde en continuité et en devenir}

Le troisième musée d'État créé en 1984 par le gouvemement du Québec est ouvert au public depuis octobre 1988. Comme le stipule le document Mission, concepts et orientations du Musée de la civilisation, wle Musce se veut d'abord un apport et un soutien à ceux et à celles qui s"intéressent aux phénomènes de civilisation. Lieu de plaisir, de détente, de réflexion, de connaissance, d'étonnement, le Musce de la civilisation veut permettre aux visiteurs d'établir un rapport critique avec l'histoire et la culture du Québec. Il veut également lui donner une connaissance du passé qui lui fournira des éléments de lecture et d'analyse des problèmes présentset peut-être même des solutions pour l'avenir. Pour ce faire, le Musée rend accessible et fait connaître un précieux héritage qu'il eclaire par la science et la mise en valeurs ${ }^{(1)}$.

Par ailleurs, il fut précisé récemment que ale Musée de la civilisation existe d'abord et avant tout pour sa clientèle qui est multiple et dont les atientes sont variéess (2). Si le Musée de la civilisation est d'abordet avant tout au service des Québécoiss ${ }^{(3)}$ ill est devenu, depuis son ouverture au public, une institution d'envergure intemationale. Sanotoriétés'est répandue jusqu'aux États-Unis et mểme en Europe et en Asie comme en témoignent plusieurs articles parus dans de nombreux journaux et revues internationaux ainsi que l'accueil de visiteurs-touristes et de journalistes de partout dans le monde.

Madame Marilyn Johnson est responsable du marketing et de la promotion touristique. Service des relations publiques et des communications au Musée de la civilisation, Quebec.

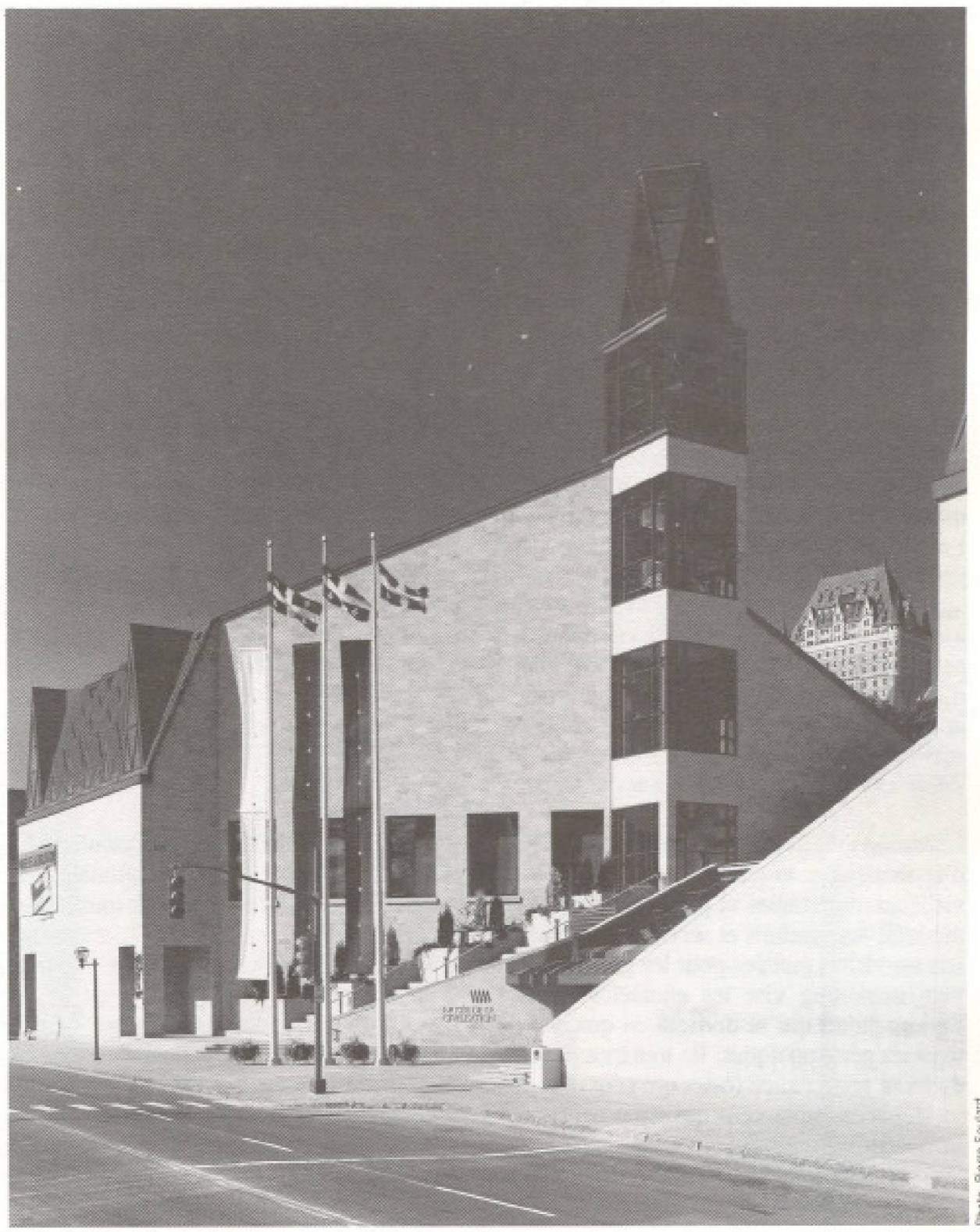

Prise de vie exterieure du Maske. Muske de la civilisation. Juilla 1991.

\section{Les besoins et attentes} des visiteurs du Musée

Les besoins et attentes des visiteurs étrangers sont ă la fois les mêmes et à la fois différents de ceux des visiteurs-touristes régionaux. Le visiteur-touriste de l'étranger et même celui de l'extérieur de la région ou de la province vient visiter le Musée par intếrêt et souvent parcuriosité. Dans le mesure où il s'agit d"un visiteur de passage, son besoin de comprendre est à certains égards plus grand que le visiteur régional. Le visiteur-touriste s'attend à ce que son hôte l' impressionne etattire rapidement sa curiosité. Comme il en est souvent à sa première visite et possiblement a la seule qu'il a l'intention de faire, ses exigences de satisfaction sont particulièrement élevées. 
Le visiteur régional, quant à lui, sait qu"il peut toujours revenir visiter le Musée, qu'il lui est possible de répéter l'expérience. Les explications en détail du concept et de la façon de faire du Musée sont moins utiles. Pair ailleurs, l'affichage dans le Musee est dins sa langue. Il est chez lui. Toutefois, en tenant compte de ces différences, le Musce tient à ce que la qualité de l'accueil soit la même pour tous ses visiteurs. D’ailleurs, «Le Musée devra faire des choix difficiles pour demeurer fidele à ses orientations. II les fera en pensant aux visiteurs d'ici et d'ailleurs qu'il doit desservirs ${ }^{(4)}$.

\section{Le Musée de la civilisation : un attrait à la fois culturel et touristique}

Le Muscede lacivilisation est a la fois un lieu de culture et un attrait touristique. Il se positionne avantageusement comme attrait touristique dans la région de Québec. Il oeuvre à développer des produits et services et à améliorer des services existants ainsi que la mise en marché des produits en fonction de l'evolution des goutts, des besoins, des préférences et des comportements des visiteurs-touristes. A cette fin, le Musée a crét un poste de marketing et de promotion touristique qui a comme raison d'être principale d'augmenter le nombre de visiteurs-touristes au Musée et de promouvoir les produits et services du Musée auprès des clientèles de l'extếrieur de larégion immédiate de Québec.

LeMusces sest doté d'un plan marketingafin d'abord de acerner les besoins et attentes des visiteurs touristiques et par la suite de promouvoir les produits et services appropriés tels les visites guidées pour les groupes. Ce plan marketing vise les clientèles touristiques adultes qui se divisent en quatre catégories géographiques: les touristes captifs de toute provenance (ceux qui sont déjà en ville, par exemple, ceux qui séjournent dans les hôtels de larégion), les touristes canadiens (hors Québec), les touristes américains et les touristes d'outre-mer.

Ces clientèles sont rejointes par des moyens de communication tels:

La diffusion d'informations et de dépliants du Musée dans les bureaux de renseignements touristiques.

Le placement de publicités dans les guides et revues touristiques.

- Le développement de forfaits avec des hôtels de la région.
La placement de publi-reportages dans des revues spécialisées.

La participation aux bourses et salons touristiques qui sont organisés par les différents intervenants touristiques, soit: les ministères du Tourisme du Québec et du Canada, l'Office du tourisme et des congrès de la Communauté urbaine de Québec, l'Association des propriétaires d'autobus du Québec, le National Tour Association, ainsi que le American Bus Association.

La participation aux tournées de familiarisation qui sont organisces à l'intention des transporteurs en autocars, des voyagistes, ainsi que des multiplicateurs de groupes québécois, canadiens, amćricains et européenss ${ }^{(5)}$.

Dans toutes les promotions et publications du Musée, que ce soit au Québec ou à l'extérieur, nous tenons compte de ce qu'écrivait José Seydoux dans son oeuvre intitulé Accueil d'aujourd'hui et de demain, aL'offre doit être décrite de façon honnête et précise afin que le client potentiel ne se fasse pas de fausses idees... $x^{(6)}$.

\section{Le Musée et ses clientèles}

Le Musée de la civilisation accueille en moyenne 2300 visiteurs par jour; soit plus de 750000 visiteurs par année. Ainsi, depuis son ouverture, plus de 2700000 visiteurs ont franchi ses portes.

Trois types de clientẻles fréquentent le $\mathrm{Mu}$ sée: une clientèle locale et régionale, une clientèle scolaire et une clientèle touristique.

\section{La clientèle locale et régionale}

Cette catégorie de visiteurs au Musce wconstitue la base de fréquentation du $\mathrm{Mu}$ sce... et pour s'assurer de la participation régulière de ses usagers, le Musce offre un choix d'expositions et d'activites à caractère temporaire car ces usagers visitent le Musée en moyenne deux fois par années 77 .

\section{La clientèle scolaire}

Le Muśé rejoint toutes les écoles primaires et secondaires de la province et offre à leurs élèves des activités éducatives et culturelles telles des ateliers et des parcours spécialement élaborés poư eux. Cette clientèle est également composée de milliers d'étudiants en provenance de l'extérieur de la province et des États-Unis.

\section{La clientèle touristique}

Principalement de langue française mais de plus en plus de langue anglaise et d'autres langues telles le japonais, l'espagnol et l'allemand, cette catégorie de visiteurs occasionnels et souvent saisonniers exige un accueil un peu différent des autres catégories de visiteurs compte tenu des differences de culture et de langue. Toutefois, comme nous l'avons dit, la qualité de l'accueil demeure aussi élevé que pour les autres groupes de visiteurs.LeMusce doit doncen tenircompte dans sa structure d'accueil ainsi que dans les produits et services qui sont offerts.

\section{La structure d'accueil du Musée}

L'accueil au Musée de la civilisation se fait par des personnes. Il existe cinq points possibles pour l'accueil au Musce. Soit:

- Les guide-animateurs à la porte d'entree;

- Les préposés aux renseignements au comptoir d'accueil;

- Les préposés à la billetterie;

- Les guides-animateurs dans les salles d'expositions;

Les gardiens de securité.

Tous les guides-animateurs du Musée sont bilingues et certains sont polyglottes. Il en va de même pour les préposés aux renseignements au poste d'accueil, pour le personinel delabilletterie, ainsique pour tout lepersonnel qui est en contact direct avec le public. we guide à l'accueil reçoit le visiteur dans le Musée, aide le visiteur à s'orienter dans le Musce et dans les salles d'expositions, informe le visiteur brievement du contenu des expositions et des activités pédagogiques et culturelles qui sont disponibles, répond a toute question - dans la mesure du possible concernant le Musée etses activités, saisit les attentes des clienteles et leur propose une programme de visite qui répond à leurs intérêtș ${ }^{(0)}$.

we guide souhaite la bienvenue aux visiteurs etchercheàcréer un climat favorableau bon déroulement de leur visite. Un accueil de qualité est déterminant pour la réussite de la visite au Musée. Le visiteur mieux situé dans l'espace pourra bénéficier davantage de sa visite. Par une information pertinente, le visiteur aura une plus juste vision de l'institution. Des documents d'information présentant et expliquant brièvement les expositions et les activités culturelles et éducatives sont remis par les guides à chacun des 
visiteurs. Les fonctions d'accueil et d'information marquent toutes les autres fonctions des guides. Ceux-ci doivent être conscients que souvent le visiteur identifie le Musée à l'accueil qui lui est réservé et à la qualité de l'information qu'il reçoit. Le guide àl'accueil doit donc être conscient des exigences qui entourent son travail auprès du public ${ }^{(5)}$.

L'accueil au Musce se traduit aussi dans la signalisation par des pictogrammes qui indiquent les différents services disponibles: les ascenseurs, les toilettes et le vestiaire, l'accès pour les personnes en chaise roulante, la boutique, etc.

\section{Comment tenir compte des touristes dans l'accueil au Musée?}

Pour assurer la même qualité d'accueil à tous, il y a lieu toutefois de tenir compte des besoins, par exemple, des visiteurs-touristes anglophones et allophones qui sont de plus. en plus nombreux. Le Musé, comme institution d'État a cependant des contraintes légales quant à l'affichage. Les lois du gouvemement du Québec portant sur la langue française nous obligent à afficher à l'extérieur et à l'intérieur du Musée en français uniquement. Après avoir constaté certaines difficultés vócues par la clientèle touristique anglophone et allophone, le Musce a notamment installe des panneaux d'information multilingues à l'entrée du Musée. Ces panneaux présentent les expositions en cours, annoncent les principales activités éducativeset culturelles offertes ainsi que les services disponibles.

De plus, puisque tous les panneaux et vignettes dans les salles d'expositions sont unilingues français, le Musce met a la disposition de ses visiteurs anglophones plusieurs exemplaires d'un English guide-book. Ces documents reprennent intégralement ou en partie le contenu des panneaux et vignettes des expositions. Il sont placés à l'entrée de chaque salle d'exposition. Par ailleurs, un guide-animateur bilingue est sur place dans la plupart des salles d'exposition pour répondre aux questions de tous les visiteurs.

\section{Comment visiter le Musée de la civilisation?}

Les visiteurs ont le choix quant aux différentes façons de visiter les expositions du Musée soit: visites libres ou visites commentées en groupe, ex.. les Visites Horloges, ou les visites générales du Musce, ex. Une heure au Musée.
Visites Libres - les visiteurs de langue française ne desirant pas de visite guid6e peuvent visiter les expositions librement sans difficulté. Leurs homologues de langue anglaise peuvent egalement visiter les expositions librementensuivantles English guide-books.

- Visites Horloges - il s' agit là de visites commentees des expositions en françis et en anglais offertes selon un horaire varié. Les visiteurs-touristes peuvent se joindre aux groupes qui se forment à l'entrée des salles d'expositions. L'horaire de ces visites est affiché près du poste d'accueil et de la billetterie du Musee.

* Une Heure au Musée - Cette visite spéciale du Muséc aété conçue particulièrement pour les visiteurs-touristes. II s"agit d"une courte présentation générale du Musée, de son conceptet de son architecture, suivi d'une brève visite d'une ou de plusieurs expositions. Cette formule permetaux visiteurs d'avoir un aperçu de ce qu'est le Musée et de choisir des expositions qu'ils désirent visiter ou revisiter plus en détail à la fin de l'activité. Sauf pendant la saison estivale, où l'achalandage ne permet pas de faire des visites guidées de groupes dans les salles d'expositions, les groupes peuvent réserver une visite guidée à l'avance afin de se garantir la disponibilité d'un guide-animateur.

\section{L'été au Musée}

Fidele à son conceptet à ses orientations, «le Musée a choisi de traiter des thèmes de façon multicommunicationnelle et d'avoir une approche souple, variée et de qualitéen matiẻre de communication avec le publico ${ }^{(9)}$. A cette fin, le Musée organise une panoplied'activités telles les ateliers et parcours spéciaux pour les jeunes et les familles, des films ainsi que des conférences selon les themes des expositions. Cesactivites sont presque toutesoffertes gratuitement aux visiteurs du Musée.

Par ailleurs, chaque été, le Musée développe autour d'une même thématique des activités culturelles et d'animation à l'intention de tous les visiteurs. Pour l'été 1992, par exemple, cette thematique explore Les woies du fleuve. Ces activités (concerts, pièces de theâtre, spectacles, danse, etc) se déroulent dans la cour intérieure du Musée et à la place Royale puisque le Musée de lacivilisation est également responsable de l'animation et de l'interprétation de la place Royale, berceau de I'Amérique française en Amérique du Nord. Les guides-animateurs affectés aux différents sites a la place Royale et à la Maison Chevalier accueillent plus de 300 000 visiteurset touristes par année. Ils offrent graluitement des visites commentees et de l'animation durant toute la saison estivale.

\section{Conclusion : \\ la qualité de l'accueil au Musée}

Au Musée de la civilisation, la qualité du service à la clientèle n'est pas une politique, mais une obsession organisationnelle qui revient un peu partout dans nos texteset dans tous nos plans; une orientation permanente qui s'est intégrée à la gestion ordinaire du Musée. Notre taux de fidelisation s"aocroit. En juillet 1991, 21\% de nos habitués en étaient à leur troisième visite dans l'annê, et $13 \%$ en étaient à leur quatrième, cinquième et sixième visite durant la même période. Nos sondages confirment des taux exceptionnels de satisfaction de la clientele (de l'ordre de $80 \%-90 \%$ et plus) et ce, sur tous les eléments que nous prenons soin de vérifier: que ce soit l'accueil, notre service de visites guidées, le contenu denos expositions, nos activités d'animation pour les jeunes et les familles. Que ce soit pour le vestiaire, le Café du Musée ou la Boutique, tous les résultats nous indiquent que nos visiteurs sont heureux de ce qui leur arrive au Musee. Mais surtout, tout ce qui arrive aux visiteurs du Musée de la civilisation commence par l'accueil chaleureux de nos guides-animateurs.

Finalement, la qualité de l'accueil au Musée de la civilisation est aussi importante que la qualitế des expositions et des activités éducatives et culturelles. Tout le personnel, des guide-animateursă l'entróedu Musće etdans les salles d'expositions, des gardiens de sécurité, jusqu'à la direction duMuséc, en est conscient et oeuvre continuellement à ce que l'accueilévolueselon les besoinsetles attentes de tous nos visiteurs.

\section{NOTES ET REEFÉRIENCES}

(1) Roland Arpin, Mission, concopts of orientations. Musbe de la civilisation, aout 1587, p. 9.

(2) Fland Arpin, Plan diructeur 1992-1995, Musbe de la civlisation, fevrier 1992, p. 14.

(3) Fialand Arpin, Mission, concepts et orientations, p. 17 .

(4) PolandAmin, Mission, conceptsotorientations, $p$. 7 .

(5) Marilyn Johnson. Plan marketing 1991-1992, Musté de la ovisstion, féwner 1991.

(1) Jose Seydoux. Accued df aujourd hui et de demain, Denges, 1984, Editions Delta \& Spes SA, Ch. 1026 Denges.

(7) Roland Arpin, Plan drecteur 1902-1995

(b) Pauline Beaudin, Aide Mámołrelguideranimateur a Iaceued). Mustes de la comlisation, jun 1992.

(9) Ricland Aspin, Mission, concept of oriontations, p. 9. 